\title{
Modeling Deep Ocean Convection: Large Eddy Simulation in Comparison with Laboratory Experiments
}

\author{
S. RAASCH AND D. ETLING \\ Institut für Meteorologie und Klimatologie, Universität Hannover, Hannover, Germany
}

(Manuscript received 9 September 1996, in final form 9 December 1997)

\begin{abstract}
A large-eddy simulation model (LES) has been applied to study deep convective processes in a stratified ocean driven by the energetic cooling at the ocean surface. Closely related to a recent laboratory experiment, the numerical experiment deals with the inverted problem of the growth of a convective mixed layer driven by a localized source of bottom heating in a rotating, stably stratified fluid. In general, good agreement is found between numerical and laboratory results.

After onset of the heating a well-mixed layer forms above the heated circular surface. Although small-scale turbulence quantities like rms velocities and length scale can be best described by the nonrotating turbulent velocity and length scales, they are also found to differ significantly from a nonrotating control run, which indicates that rotation affects but does not control the turbulence.

Due to the horizontal radial temperature gradient between the mixed layer and the ambient fluid a rim current develops around the periphery of the heated surface. Its near-surface maximum can be well described by a simple thermal wind law. The strong counterrotating current also observed in the laboratory at greater heights above the surface is found to be mainly driven by surface friction and should not be observed in the ocean. As time progresses, the rim current becomes unstable, eventually generating a field of baroclinic eddies that stop the mixed layer growth by causing some horizontal exchange between the convective layer and its cooler surrounding. The wavelength of the instabilities slowly increases with time and is clearly related to the local Rossby radius.
\end{abstract}

\section{Introduction}

The problem of deep oceanic convection induced by localized surface cooling has received considerable attention in the last years. Results from field observations (e.g., in the Greenland Sea or the Gulf of Lions), laboratory experiments, and numerical simulations have led to some theoretical predictions concerning the structure of the convective region like plume scale, chimney scale, and rim current by, for example, Klinger and Marshall (1995), Send and Marshall (1995), and Visbeck et al. (1996). The comparison of these scaling arguments with real ocean data on deep convection is somewhat restricted due to the lack of detailed measurements of convective plumes and chimneys, although field experiments have provided very impressive cases of deep ocean convection (e.g., Morawitz et al. 1996; Schott et al. 1996).

As deep ocean convection is not easily observed in the field, various laboratory experiments have been performed in order to understand the physical processes

Corresponding author address: Dr. Siegfried Raasch, Institut für Meteorologie und Klimatologie, Universität Hannover, Herrenhäuser Str. 2, 30419 Hannover, Germany.

E-mail: raasch@muk.uni-hannover.de involved in this phenomenon. One of the key questions that has to be resolved is the influence of the earth's rotation on deep convection. Hence, several laboratory experiments have been performed on convectively driven turbulence in rotating homogeneous fluids (e.g., Boubnov and Golitsyn 1990; Fernando et al. 1991; Brickman and Kelley 1994; Maxworthy and Narimousa 1994; Brickman 1995). These experiments show that there is a transition at a critical distance $h_{c}$ from the buoyancy source where the flow field changed from fully developed $3 \mathrm{D}$ turbulence close to the source to quasi-2D rotationally controlled vortex structures with axes parallel to the rotational axis. Maxworthy and $\mathrm{Na}-$ rimousa (1994) found that this transition depth $h_{c}$ was greater than typically observed convective layer depths in the ocean and often greater than the depth of the ocean.

Inspired by these results, recently Coates et al. (1995) and Ivey et al. (1995) performed some laboratory experiments using a parameter range where rotation affects, but does not control, turbulence. They used a cylindrical rotating tank where the convective mixed layer is generated by a localized source of bottom heating, accounting for the fact that deep ocean convection is primarily generated by intense localized surface cooling in only a few high latitude locations (e.g., Gascard 
1991). Extending the work of Maxworthy and Narimousa (1994), their experiments focused on the case of an initially stably stratified fluid because oceanic observations indicate that there is always some stable background density stratification present. In these cases, after initiation of the buoyancy flux a mixed layer forms above the buoyancy source, leading to a horizontal temperature gradient between the mixed layer and the undisturbed surrounding. Due to this baroclinic situation and the background rotation a rim current develops around the heated surface, which initially confines the heated fluid to the region above this surface. After some rotational periods this rim current becomes unstable, generating a field of baroclinic eddies that lead to a lateral exchange of heated and ambient fluid and arrest the mixed layer deepening after some rotational periods. Recent experiments on localized heat sources with a two-layer fluid (Narimousa 1996) and continuous stratification (Whitehead et al. 1996) also confirm these principle findings.

The problem of rotating convection with possible applications to the ocean has been also addressed by numerical simulations (e.g., Raasch and Etling 1991; Jones and Marshall 1993; Garwood et al. 1994; Julien et al. 1996b). But these simulations are also difficult to compare with ocean data on deep convection for the same reasons valid for theoretical predictions and laboratory experiments on rotating convection. Although these models provide flow structures like plumes, chimneys, and rim currents with much detail it can be stated that they have not been verified properly against field data so far. This lack of confidence in numerical models could be improved by testing model results against laboratory experiments, which can provide more detailed measurements under controlled external parameters than would be possible in the ocean currently. If the models are verified in this way, one could be more confident in the model results if applied to real ocean situations. This is the main intention of our study presented here.

We tried to study these processes of turbulent convection over a localized source of buoyancy with background rotation as investigated in laboratory experiments with a large-eddy simulation model (LES). A number of other numerical modeling studies of deep ocean convection and the influence of background rotation on turbulent convection has been performed up to now, but in these studies convective processes have either been totally parameterized and attention was focused on large-scale flow (e.g., Madec et al. 1991) or subgrid-scale processes were parameterized by using constant eddy diffusivities (Jones and Marshall 1993; Klinger and Marshall 1994; Sander et al. 1995; Julien et al. 1996a), which makes quantitative comparisons of numerical and laboratory models diffcult (Maxworthy and Narimousa 1994). Jones and Marshall (1993) used a localized source of buoyancy but they only considered a homogenous ocean. In general, their experiments supported the laboratory studies of Maxworthy and Nari- mousa (1994) in showing the formation of rotationally controlled plumes within the convective chimney, which eventually becomes baroclinically unstable. Raasch and Etling (1991) have applied an LES model to study rotating thermal convection, but without specific application to the ocean. Recently, LES models have been used to study deep ocean convection with respect to rotation on penetrative convection (Julien et al. 1996b) and to the effect of thermobaricity on convection (Garwood et al. 1994; Denbo and Skyllingstad 1996). Main results are that rotation reduces penetration at the interface with the stably stratified layer, while thermobaric instability has just the opposite effect. These numerical studies used a horizontal homogeneous buoyancy source since they are concentrating on the initial violent mixing phase of the convective process.

Our numerical experiments are designed to investigate both small-scale turbulent convective processes and the baroclinic instability of the evolving rim current as well as the interaction between the convective chimney and the ambient fluid. They rely strongly on the laboratory experiments of Coates et al. (1995) - further on referenced as the "laboratory experiment" — especially since accurate measurements and analyses are available from these experiments, which should allow detailed comparisons between numerical model and laboratory results. Since analyses of model data can be performed in a much easier and more complete way than for the laboratory experiment, we hoped for further insight in the involved convective and baroclinic processes. Preconditioning of the density stratification by the action of wind stress prior to the start of cooling (Killworth 1983) as well as thermobaric effects (Garwood et al. 1994; Denbo and Skyllingstad 1996) have not been considered in the laboratory experiment and are hence outside the scope of our investigations.

Despite these limitations we think that the experiments by Coates et al. (1995) provide a very detailed dataset on rotating convection, from which numerical models can be verified. This is of some importance because it is difficult, at present, to compare model results with oceanic field data for deep convection. [This is somewhat better for the atmospheric convective boundary layer, where various LES models have been tested against comprehensive datasets; see, e.g., Nieuwstadt et al. (1991).] In this spirit we mention also the recent work by Lavelle and Smith (1996), who presented numerical simulations on the laboratory experiments of rotating convective line plumes performed by Fernando and Ching (1993).

\section{Description of the numerical model}

The three-dimensional LES model we used in this study is based on the nonhydrostatic Boussinesq equations and is described in detail in Raasch and Etling (1991). Subgrid-scale turbulence is modeled according to Deardorff (1980). A prognostic equation is solved 
for the subgrid-scale turbulence kinetic energy (TKE) and the subgrid fluxes are parameterized by first-order closure where the subgrid-scale eddy coefficients are related to the TKE. Similar subgrid turbulence schemes have been also used by Garwood et al. (1994) and Julien et al. (1996b). The original model was developed to study atmospheric convective boundary layers, which is one reason that we treat the inverted problem of heating a fluid from below rather than cooling from above. On the other hand, this method is also convenient in laboratory experiments and has just been used by Coates et al. (1995), so laboratory and numerical results can be compared directly. Since only time-dependent equations for momentum, temperature, and TKE are solved, the model is unable to simulate thermobaric effects, which have been included in the LES simulation of Garwood et al. (1994) and Denbo and Skyllingstad (1996).

\section{a. Boundary and initial conditions}

In correspondence with the laboratory experiments, no-slip boundary conditions have been used for the horizontal velocities at the lower boundary. It should be noted that free-slip conditions would be the more realistic conditions for the ocean surface (see, e.g., Garwood et al. 1994 or Julien et al. 1996b) but we choose no-slip conditions mainly for reasons of comparison with the experiment.

Monin-Obukhov similarity is assumed between the surface and the first computational grid point above. A constant buoyancy flux $B$ is prescribed within a circular surface located in the center of the model domain, while zero heat flux was applied outside. Constant heat flux in this case means that only the horizontal mean value over the circular surface is fixed. The values at the single grid points were changed in space and time by a Gaussian random function in order to eliminate some very regular small-scale instabilities, which otherwise would develop at the rim of the heated disk during the initial phase of the experiments. These small-scale instabilities have not been found in the laboratory experiments since a really constant uniform buoyancy flux could not be guaranteed. The diameter of the heated disk is half the horizontal extension of the model.

At the upper boundary zero heat flux and no-slip conditions are established, while horizontal boundary conditions are chosen to be cyclic. This will limit the numerical experiments to the point where the evolving and outspreading instabilities begin to interact with their cyclic counterparts.

All runs start with a stably stratified medium at rest or in solid body rotation with a constant Brunt-Väisälä frequency $N$. The principle initial model setup is shown in Fig. 1.

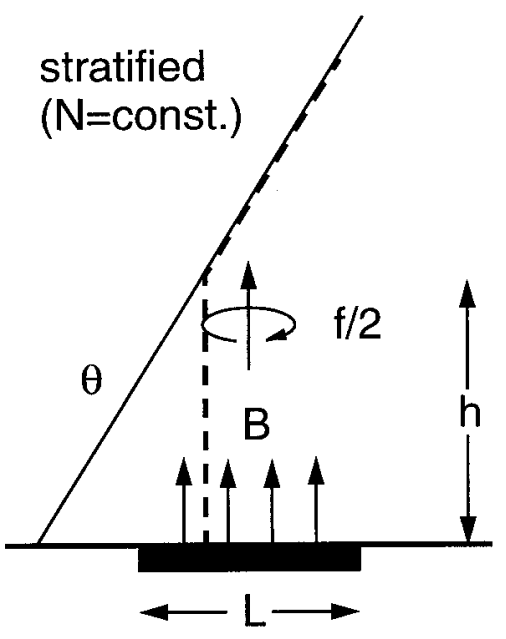

FIG. 1. Principle initial model setup. A rotating stably stratified fluid is destabilized by applying a buoyancy flux only over a part of the surface. The dashed line shows a typical temperature profile within and above the early growing mixed layer.

\section{b. Numerics}

The numerical methods are the same as described in Raasch and Etling (1991) except for the advection of scalar quantities (temperature and TKE), which is now accomplished using the monotonic scheme of Chlond (1994) based on the flux limiting scheme of Bott (1989). Grid spacing in the model is uniform with $\Delta x=\Delta y=$ $\Delta z$. We have to use a grid resolution that is able to resolve the small-scale turbulence within the evolving mixed layer. We used a grid spacing of $25 \mathrm{~m}$ with 160 $\times 160 \times 41$ grid points yielding a domain size of 4000 $\mathrm{m}$ in the horizontal and $1000 \mathrm{~m}$ in the vertical direction. The ratio of horizontal to vertical extension of the model, as well as the extension of the heated surface, is chosen the same as in the laboratory experiments by Coates et al. (1995).

The model was run on a Siemens-Nixdorf S400/10 at the Regionales Rechenzentrum für Niedersachsen (RRZN).

\section{Dynamics of deep convection}

\section{a. Uniform heat flux}

Typical properties of a convective mixed layer forced by a constant buoyancy flux $B$ at the surface $z=0$ of an initially stably stratified and nonrotating fluid are well known from the atmospheric boundary layer (e.g., Stull 1988). A turbulent mixed layer will rapidly develop and grow from the surface, for which the turbulent velocity scale is given by (Deardorff 1972)

$$
q=(B h)^{1 / 3},
$$

where $h$ as the typical length scale is the depth of the mixed layer. The mixed layer will grow not only by the surface buoyancy flux but also due to entrainment pro- 
TABLE 1. A summary of the numerical runs. The duration of the experiments can be taken from Fig. 9. The mixed layer height $h_{e}$ at the end of the runs is defined as the height, where the minimum value of the vertical heat flux occurs.

\begin{tabular}{|c|c|c|c|c|c|c|c|c|}
\hline Run & $\begin{array}{c}f \\
\left(s^{-1}\right)\end{array}$ & $\begin{array}{c}T \\
\text { (s) }\end{array}$ & $\begin{array}{c}N \\
\left(\mathrm{~s}^{-1}\right)\end{array}$ & $\begin{array}{c}B \\
\left(\mathrm{~m}^{2} \mathrm{~s}^{-3}\right)\end{array}$ & $\begin{array}{c}h_{c} \\
(\mathrm{~m})\end{array}$ & $\begin{array}{l}h_{e} \\
(\mathrm{~m})\end{array}$ & $N / f$ & $\mathrm{Ro}_{c}$ \\
\hline 0 & 0.00 & - & $1.28 \times 10^{-2}$ & $3.27 \times 10^{-3}$ & - & 650 & - & - \\
\hline 1 & 0.01 & 1256 & $1.28 \times 10^{-2}$ & $3.27 \times 10^{-3}$ & 743 & 555 & 1.28 & 0.103 \\
\hline 2 & 0.01 & 1256 & $9.04 \times 10^{-3}$ & $3.27 \times 10^{-3}$ & 743 & 670 & 0.90 & 0.085 \\
\hline 3 & 0.01 & 1256 & $1.81 \times 10^{-2}$ & $3.27 \times 10^{-3}$ & 743 & 345 & 1.81 & 0.166 \\
\hline 4 & 0.02 & 628 & $1.28 \times 10^{-2}$ & $3.27 \times 10^{-3}$ & 263 & 510 & 0.64 & 0.039 \\
\hline 5 & 0.005 & 2513 & $1.28 \times 10^{-2}$ & $3.27 \times 10^{-3}$ & 2102 & 480 & 2.56 & 0.306 \\
\hline 6 & 0.01 & 1256 & $1.28 \times 10^{-2}$ & $1.64 \times 10^{-3}$ & 525 & 360 & 1.28 & 0.111 \\
\hline 7 & 0.015 & 838 & $1.28 \times 10^{-2}$ & $1.64 \times 10^{-3}$ & 286 & 420 & 0.85 & 0.113 \\
\hline
\end{tabular}

cesses at the top of the mixed layer, where warmer fluid of the stable layer is mixed downward. Without entrainment the mixed layer will grow according to

$$
h_{t}=2^{1 / 2} B^{1 / 2} t^{1 / 2} / N \text {, }
$$

where $N$ is the Brunt-Väisälä frequency, which explains roughly $80 \%-90 \%$ of the observed variation of the mixed layer depth (Stull 1976).

In the presence of rotation with angular velocity $\omega$ $=f / 2$, a turbulent mixed layer will again grow from the surface but the convective turbulence will be affected by rotation due to Coriolis force. Rotation controls the turbulence only if the turbulent Rossby number based on small-scale properties

$$
\text { Ro }=q /(f h)
$$

is about 1 , which is the case when the eddy turnover timescale for the largest eddies in the convective layer $h / q$ is on the order of the rotational timescale $f^{-1}$. Assuming Ro $\sim 1$ and combining (1) and (3) results in

$$
h_{c} \sim\left(\frac{B}{f^{3}}\right)^{1 / 2}
$$

as the critical depth of the boundary layer.

A number of different observations (Hopfinger and Linden 1990; Fernando et al. 1991; Maxworthy and Narimousa 1994) suggest that the critical height lies in the range

$$
13\left(\frac{B}{f^{3}}\right)^{1 / 2}<h_{c}<42\left(\frac{B}{f^{3}}\right)^{1 / 2}
$$

or equally $0.2<$ Ro $<0.44$. For greater distances from the surface velocity and length scales are independent from the mixed layer height $h$ (Fernando et al. 1991). The rotational velocity scale was found to be

$$
q=(B / f)^{1 / 2} .
$$

Coates et al. (1995) showed that critical depths computed from Eq. (5) using typical values for $f$ and $B$ in the ocean are large compared to the observed depths of deep convection and even to the depth of the ocean itself. For that reason they conducted their experiments for a parameter range where the mixed layer depth is always below its critical range so that rotation does not control the turbulence and the nonrotating velocity and length scales are valid.

We proceed in the same way with our numerical experiments. A summary of the numerical runs is given in Table 1, which also shows the minimum critical depth [left side of Eq. (5)] and the maximum value of the mixed layer height reached at the end of the runs. In order to compare our results to the laboratory experiments and to ocean observations we list also the ratio $N / f$ and the convective Rossby number $\mathrm{Ro}_{c}$, defined as (e.g., Maxworthy and Narimousa 1994; Klinger and Marshall 1995)

$$
\operatorname{Ro}_{c}=\left(\frac{B}{f^{3} h^{2}}\right)^{1 / 2} .
$$

In the laboratory experiments of Coates et al., as well as in our numerical simulations, the height of the convective layer is growing in time. Hence we take the final height $h_{e}$ for calculating $\mathrm{Ro}_{c}$ from (7).

It should be mentioned that for all model results the mixed layer height is defined as the height where the vertical heat flux has its smallest negative value. It can be easily determined from the vertical profiles of heat flux (see Fig. 8). Since no heat flux was measured in the water tank experiment, Coates et al. used the theoretical definition of mixed layer height $h_{t}$ given by (2) whenever they need it in their analysis (see also discussion at the end of section $4 b$ ).

\section{b. Nonuniform heat flux}

Ivey et al. (1995) showed for the case when the buoyancy flux $B$ is applied over only a finite circular portion of diameter $L$ (see Fig. 1) that the mixed layer will develop only over the segment $L$ and will still grow according to Eq. (2). A horizontal buoyancy anomaly $g^{\prime}$ is now developed between the mixed fluid and the ambient fluid. The anomaly has its largest value,

$$
g^{\prime}=N^{2} h,
$$

at the surface and decreases linearly to zero at the top of the mixed layer, provided that the ambient fluid has a stratification with $N=$ const and that the mixed layer 

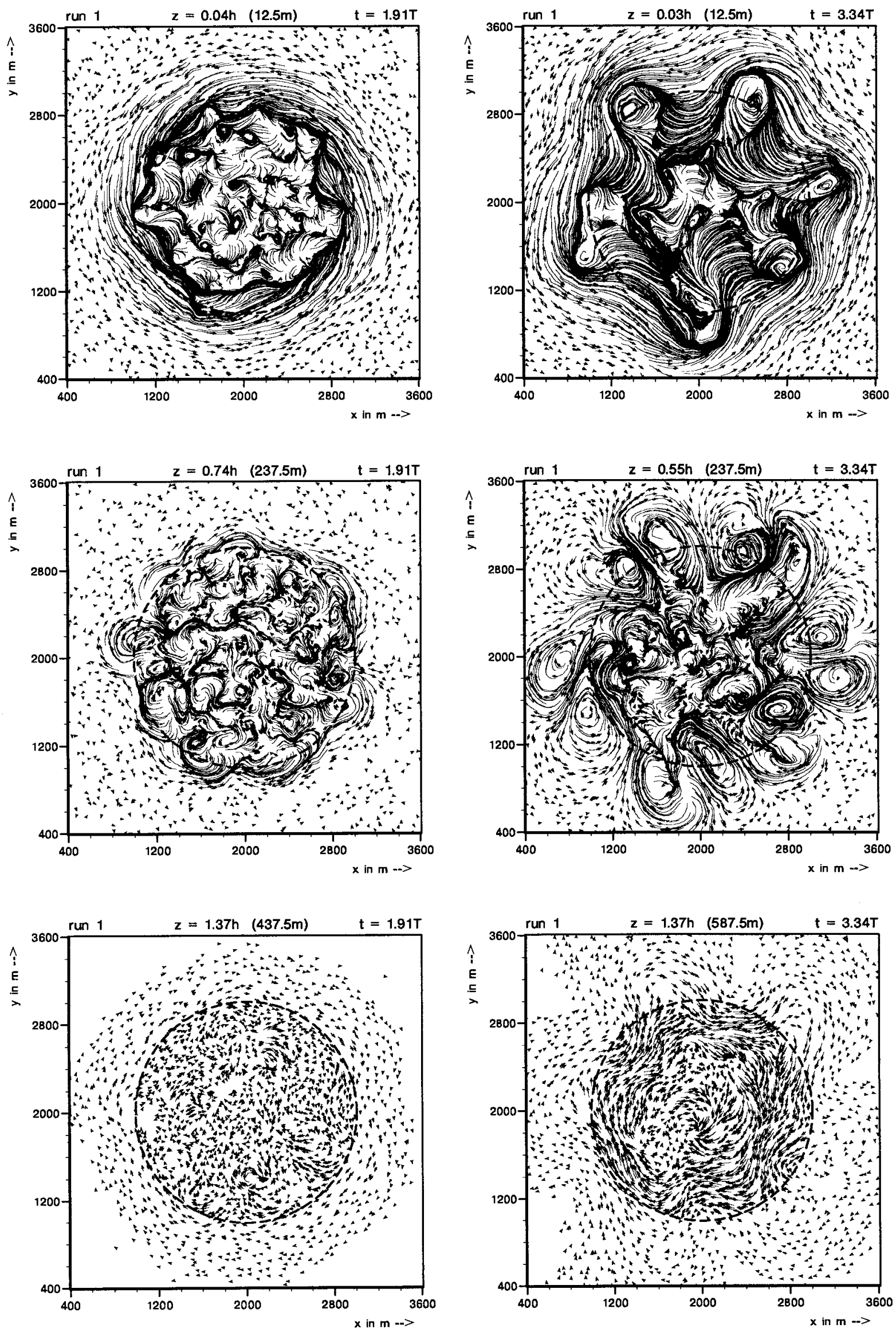
is still well mixed. This should be the case as long as Ro will stay above its critical value, which means that the rotational adjustment timescale $f^{-1}$ is large compared to the timescale for vertical mixing.

Due to the buoyancy anomaly the fluid will attempt to adjust horizontally in a timescale $N^{-1}$, but the Coriolis force limits this lateral adjustment to a scale $L_{R}$, the Rossby radius of deformation based on mixed layer height (Coates et al. 1995), defined by

$$
L_{R}=N h / f \text {. }
$$

It represents the scale at which the adjusting forces associated with the horizontal temperature gradient are balanced by the counteradjusting forces associated with rotation.

The horizontal temperature gradient across the outer boundary of the mixed layer region will cause a thermal wind described by

$$
f \frac{\partial u}{\partial z}=\frac{\partial g^{\prime}}{\partial r},
$$

where $r$ is the radial coordinate and $u$ the azimuthal velocity, which must form a rim current of width equal to the Rossby radius $L_{R}$ around the periphery of the convective region. Assuming that the buoyancy anomaly averaged over the total mixed layer depth is given by $g^{\prime}=\frac{1}{2} N^{2} h$ and substituting (8) into (10) yields

$$
u=\frac{1}{2} N h \quad \text { or } \quad u=\frac{1}{2}(B t)^{1 / 2},
$$

where $h$ is substituted from Eq. (2). This is the maximum rim current velocity that should be observed at the surface when surface friction can be neglected. With given temperature profiles for the convective region and the outer region as sketched out in Fig. 1, the rim current velocity should linearly decrease to zero at the top of the mixed layer.

Rim current width and velocity will increase with $t^{1 / 2}$, but linear stability theory (Samelson 1993) predicts that the flow is unstable to all wavelengths $\lambda>2.9 L_{R}$ and the fastest growing wavelength has the value

$$
\lambda \approx 5 L_{R} \text {. }
$$

Coates et al. pointed out that some assumptions made by the linear stability theory are not fulfilled by the flow. For example, the base flow is not steady (i.e., $L_{R}$ is not constant since mixed layer height increases with time), and the initial disturbances are not equally distributed over the whole wavenumber space but have a preferred range with scales imposed by the convective turbulence.

\section{Results from the model}

We have conducted a number of numerical runs in order to analyze the influence of three main parameters, that is, rotation rate $f$, surface buoyancy flux $B$, and background stratification $N$. They are summarized in Table 1, which also includes the rotational period $T=$ $4 \pi / f$, the minimum critical height $h_{c}$ [left side of Eq. (5)], and for comparison with the experiments, $N / f$ and $\mathrm{Ro}_{c}$. We might note here that Coates et al. (1995) performed their experiments in the range $0.59 \leq$ N/f $\leq$ 2.0 and $0.047 \leq \mathrm{Ro}_{c} \leq 0.193$. The mixed layer height $h_{e}$ (see Table 1 ) at the end of nearly all runs stays below the corresponding critical height so that rotation must not control the turbulent convection. The model runs 4 and 7 exceed the critical level but they stay within the range of heights given by Eq. (5).

As already indicated earlier, our LES model was designed for simulations of the atmospheric convective boundary layer. Hence, in the present simulations we do not model the laboratory experiments directly by choosing physical properties of the working fluid and dimensions of the setup as in the laboratory. Rather, we investigate a model atmosphere with parameters tuned to the experiments. The same procedure has already been used in earlier simulations (Raasch and Etling 1991), where data of the laboratory experiments by Fernando et al. (1991) were used for comparison. The ratio $N / f$ and the convective Rossby number $\mathrm{Ro}_{c}$ have especially been taken in the range of the experiments (see above), and the relative size of the computational domain to the size of the heat source has been taken accordingly.

With respect to the results, we have normalized most figures in the same way as in the laboratory experiments so that a direct comparison is possible. In some cases, dimensional units have been used, which should not be compared to real ocean data but rather be taken as a model atmosphere comparable to the laboratory experiments.

\section{a. Typical flow field}

Before we focus on the turbulent structures of the mixed layer and the evolving baroclinic instabilities, we would first like to present some flow and temperature fields from a typical run. Figure 2 shows horizontal cross sections at two different times and for three different heights respectively. The heated area is marked by a dashed circle. Plotted streaklines are derived from the horizontal velocity components by advecting weightless

$\leftarrow$

Fig. 2. Horizontal cross sections of the horizontal velocity from run 1 for various heights at time $t=1.91 T$ (left column) and $t=3.34 T$ (right column). Only the central part of the computational domain is shown. The heated area is marked by a dashed line. Streaklines are produced by advecting weightless particles in the instantaneous velocity fields over a time interval of $10 \mathrm{~min}(0.5 T)$, and flow direction is represented by arrows at the tip of the streaklines. 


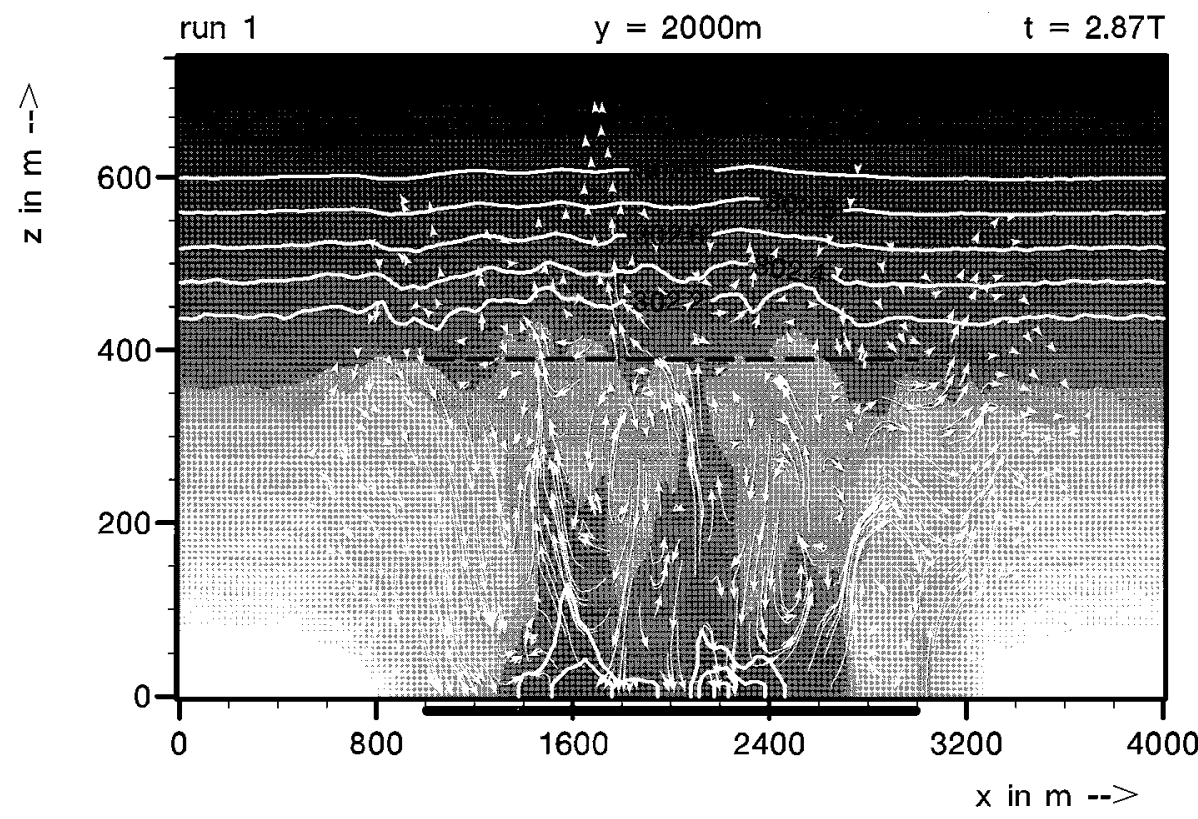

FIG. 3. Vertical cross section of the temperature field. The section is through the center of the heated surface located at $x=2000 \mathrm{~m}$. A thick black line indicates the heated surface extending from $x=$ 1000 to $x=3000 \mathrm{~m}$. Low temperatures are marked by light gray scale, high temperatures by dark gray scale. In addition, isolines of temperature are included between $z=400$ and $z=600 \mathrm{~m}$. The black dashed line indicates the mean depth of the mixed layer. Streaklines are obtained by using only the $x-$ $z$ components of velocity. Particles are advected over a time interval of $0.16 T$.

particles in the instantaneous flow fields for a limited time. The flow direction is represented by arrows at the tip of the streaklines.

At time $1.91 T$ a pronounced circular current has already developed near the surface at the rim of the heated surface (upper left picture in Fig. 2). Like the tank rotation the rim current has an anticlockwise direction, which has to be expected since inflowing fluid is turned to the right-hand side by Coriolis force. Due to surface friction there is still a net inflow that is stopped at a strong convergence line where the fluid is forced upward. For reasons of continuity there must be a corresponding outflow at greater heights that now must be forced in a clockwise direction. This behavior can, in fact, be observed in the midleft picture of Fig. 2 at $0.74 h$. The clockwise motion of the fluid even persists, although much weaker, at heights above the mixed layer (lower left panel).

The near-surface rim current separates a quiescent far field from the convective regime above the heated surface characterized by some small-scale structures and regions of convergence and divergence that are typical for the surface region of the nonrotating CBL (see, e.g., Raasch and Etling 1991) and forced by the convective updrafts and corresponding downdrafts. The convective motions dominate the flow field at $0.74 h$, and even at $1.21 \mathrm{~h}$ weaker small-scale motions appear over the heated region caused by convective plumes penetrating into the stable layer.

Penetration of plumes is clearly evident in Fig. 3, which shows a vertical cross section through the center of the heated surface at $2.87 T$. This figure also illustrates that the convective plumes may extend through the whole depth of the mixed layer and that at this time no significant horizontal exchange of fluid takes place between the mixed layer and its surrounding.

The upper right panel of Fig. 2 taken at 3.34T shows that at this time the rim current has already become unstable, forming larger-scale rotationally induced instabilities around the periphery of the heated region. The small-scale turbulence is now strongly influenced by the growing eddies and can be only weakly identified near the center of the heated region. At $0.55 h$ the wavelike structure of the rim current has turned into dipolelike vortex pairs that now make some heavy exchange between the heated and ambient fluid. The clockwise flow above the mixed layer (lower right picture at 1.37h) now extends over most parts of the heated area except at its center. This is purely an effect of the thermal wind. Since the surfaces of constant temperature within the inversion above the heated area are pushed upward (see Fig. 3), the horizontal radial temperature gradient, and thus the density gradient, has an opposite direction compared to the near-surface region that must result according to Eq. (10) in opposite flow directions. At later times the near-surface baroclinic waves divide into single vortices, which spread out over the whole computational domain.

Before proceeding into a more quantitative discussion of the model results we would like to focus once again 

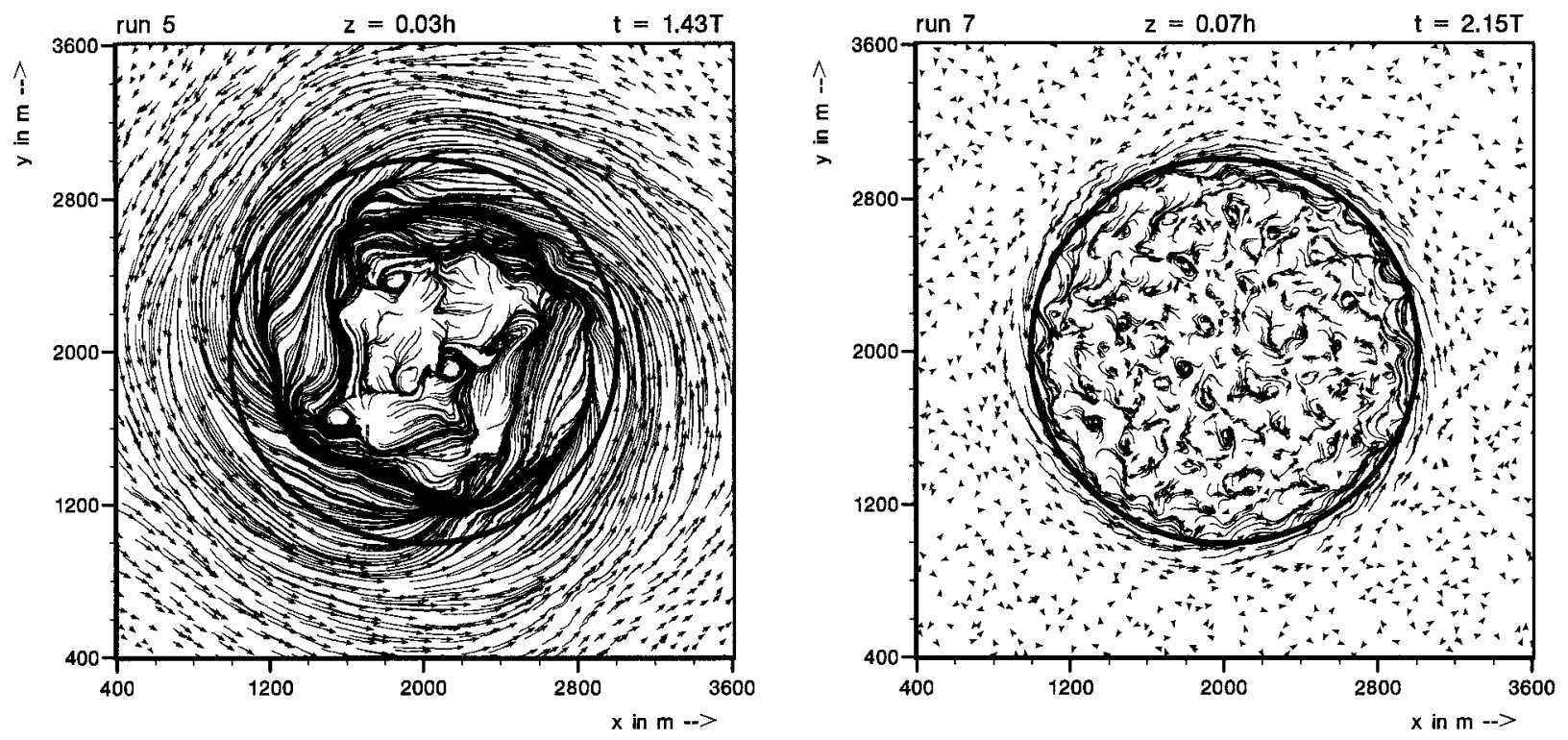

FIG. 4. Near-surface horizontal streaklines from run 5 (left) and run 7 (right). In both cases, particles have been advected over a time interval of $10 \mathrm{~min}$.

on the influence of surface friction on the near-surface flow. It should be mentioned that we used the same surface roughness length $z_{0}$ in all our runs. Figure 4 shows horizontal cross sections of the near-surface flow from the two model runs with different rotation rates. A low value of the Coriolis parameter $f$ means that surface friction becomes stronger compared to the Coriolis force and this results in a stronger inflow (left panel of Fig. 4). On the other hand, large values of $f$ reduce the influence of surface friction and cause much less inflow (right panel of Fig. 4). This behavior will become important when we discuss the vertical profiles of maximum rim current velocities in section 4c.

It should also be pointed out that all the flow structures described above have also been found in the laboratory experiment (see, e.g., Figs. 2, 4, and 6 of Coates et al. 1995) and that there is an excellent qualitative agreement between the numerical and laboratory results.

\section{b. Effect of rotation on turbulent convection}

In this section we will analyze the convective turbulence above the heated surface in detail. Coates et al. (1995) found from their laboratory experiments that nonrotating scaling with $(B h)^{1 / 3}(1)$ gives a much better description of their data than rotational scaling (6). Figures 5 and 6 show model results of root-mean-square vertical and horizontal velocities normalized by nonrotational (Fig. 5) and rotational scaling (Fig. 6) plotted against nondimensional height. All profiles presented in this section are horizontally averaged over the heated circular surface except in those regions already influenced by the rim current. They are also averaged in time over a period of about $T / 4$ and taken for times when the convective mixed layer has reached a quasi-steady state and when the rim current was still stable.

In principle, horizontal and vertical rms velocities normalized by the nonrotational velocity scale (Fig. 5) are quite similar to those found in the laboratory experiment. The vertical component has its maximum value at about $0.3 h$, typical for the nonrotating boundary layer (Schmidt and Schumann 1989; Deardorff and Willis 1985), which is also confirmed by the nonrotating model run 0 . Figure 5 shows that the rms vertical velocity from this nonrotating run compares quite well with results from a laboratory experiment of convection between two rigid plates by Adrian et al. (1986), although larger deviations occur at the top of the mixed layer since in the model it is terminated by an inversion rather than a rigid lid. The profiles determined from the rotating runs have a similar shape but their maximum values decrease with increasing rotation. This agrees with results from earlier numerical experiments by Raasch and Etling (1991) or Jones and Marshall (1993) showing that rotation significantly decreases vertical velocity variances. The laboratory experiment (see Fig. 8 in Coates et al. 1995) also yields a reduced rms vertical velocity with a depth-averaged value of $0.3(B H)^{1 / 3}$, comparable to our run 4 but with maximum values at greater heights of about $0.6 h$. The laboratory data are all taken for the same value of $f$ and therefore they cannot be used to determine the influence of a varying rotation rate. The numerical experiments do show this influence and also show, as expeceted, that other parameters like surface buoyancy flux or initial buoyancy frequency do not control the rms vertical and horizontal velocities normalized by $(B H)^{1 / 3}$.

Figure 6 is based on the same data as Fig. 5 but now 

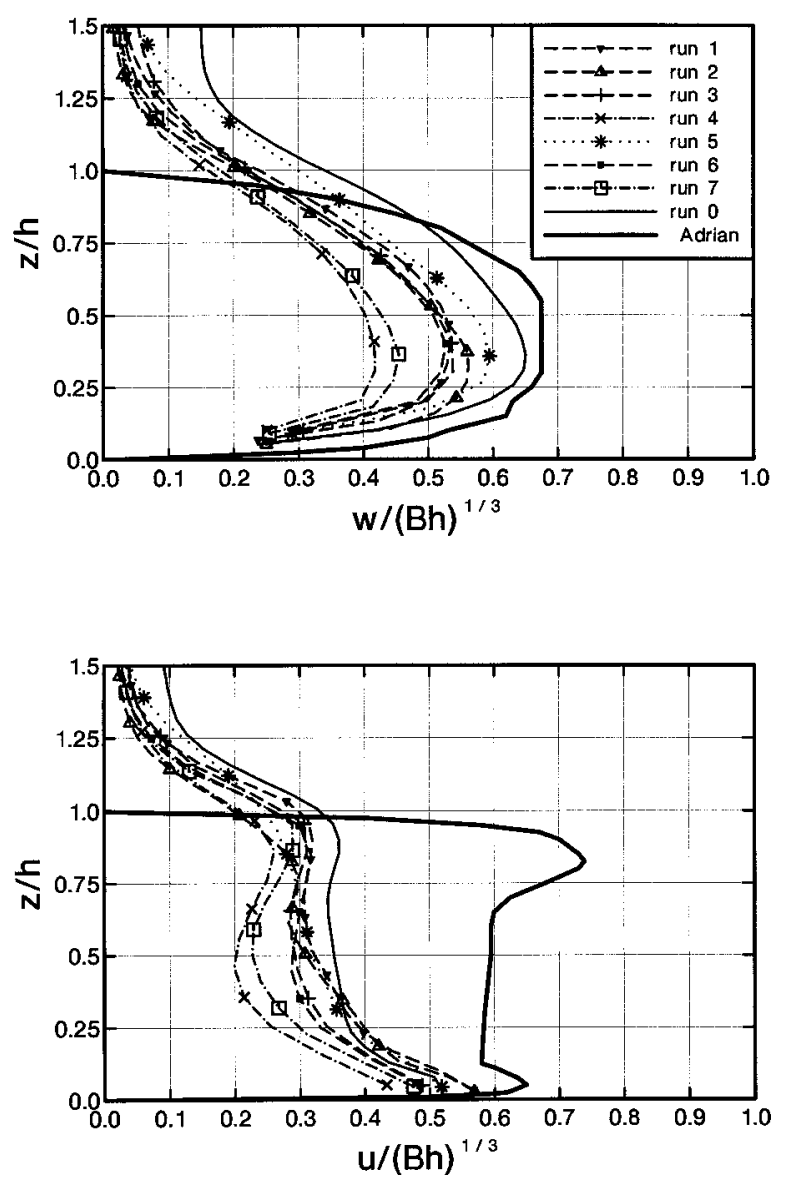

FIG. 5. Root-mean-square vertical (upper) and horizontal (lower) velocities normalized by the nonrotational velocity scale $(B h)^{1 / 3}$ and plotted against nondimensional height. Lines from the same run are marked identical in both graphs. The fat solid lines are the experimental observations of Adrian et al. (1986). The thin solid lines are from the nonrotating control run.

velocities are normalized using the rotational scale $(B /$ $f)^{1 / 2}$. Also included are the results from laboratory experiments by Fernando et al. (1991). It is evident that the difference between the numerical results and this laboratory experiment is much larger than between the profiles obtained by nonrotational scaling and the experimental results of Adrian et al. (1986). The laboratory data of Coates et al. (1995) show normalized values between 0.4 and 1.3 for the vertical component and between 0.3 and 0.8 for the horizontal component. These values fall well within the range predicted by the numerical model, confirming that the model is running in a parameter range similar to the laboratory experiment. From the model data we reach the same conclusion as Coates et al. (1995), that is, that for the investigated parameter range nonrotational scaling gives a much better description of the data. Nevertheless, Fig. 6 illustrates, like Fig. 5, that an increasing rotation effects the vertical profiles of the rms velocity components, and although it is not as evident as in Fig. 5, there is a
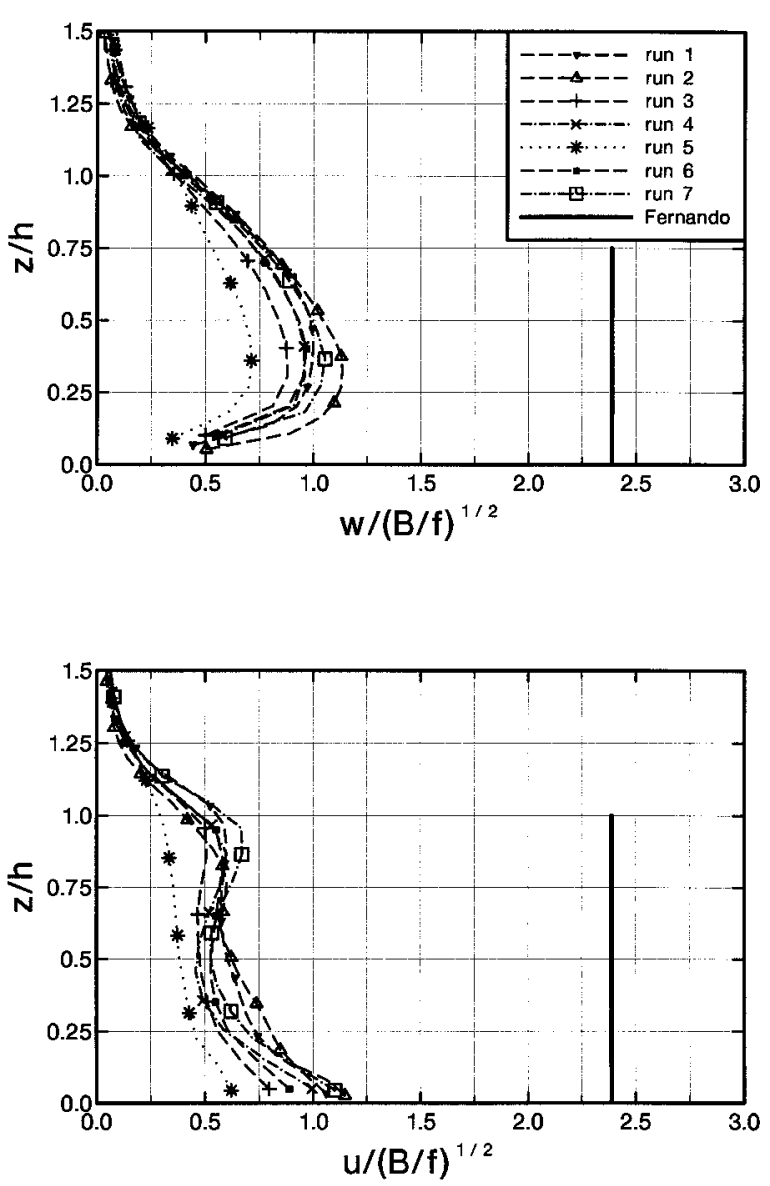

FIG. 6. A plot of the same rms velocity data as in Fig. 5 but now nondimensionalized with the rotational scale $(B / f)^{1 / 2}$. The fat solid lines are the experimental observations of Fernando et al. (1991).

tendency that runs with larger values of $f$ are closer to the regime of rotationally controlled convection.

It is known from earlier investigations (e.g., Raasch and Etling 1991) that increasing rotation reduces all large-scale motions, so the integral length scale $d$ of turbulent convection should be decreased with increasing value of $f$. Coates et al. (1995) found an estimated mean value of $d \approx(0.38 \pm 0.06) h$ by measuring the horizontal distance between the convective plumes at midheight from dye images and streak photographs. This value is somewhat larger than $d \approx 0.25 h$ found by Hunt (1984) and Adrian et al. (1986) for a homogeneous nonrotating fluid, which, however, might be difficult to apply since there is a stable background stratification present in the laboratory experiment as well as in our numerical experiment. The numerical model allows one to calculate the turbulent length scale from the autocorrelation function of the vertical velocity component and to examine how it depends on height (see Fig. 7, where all variables are normalized with the mixed layer height). We analyzed three runs with medium, large, and no rotation. The depth-averaged values for $d$ within the mixed layer are about $0.42 h$ for run 0 (no rotation), 


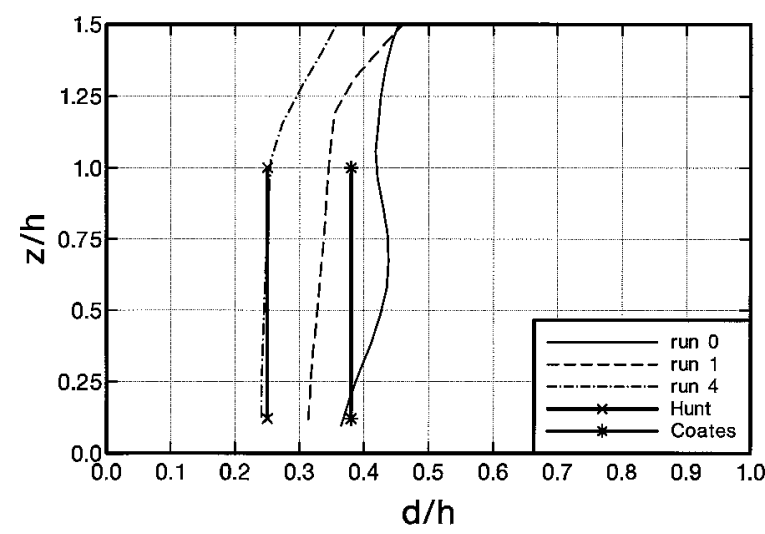

FIG. 7. Turbulent length scale plotted against height, both normalized with mixed layer height $h$. Fat solid lines are experimental observations of Hunt (1984) (X) and Coates et al. (1995) (*).

$0.33 h$ for run 1 (medium rotation), and $0.25 h$ for run 4 (large value of $f$ ). These values are similar to the observed one but (once again) the numerical model indicates a decrease of the turbulent length scale with increasing rotation, which means that larger-scale motions are reduced. This is in agreement with other laboratory studies of rotating convection (e.g., Boubnov and Golitsyn 1990). In the nonrotating case the turbulent length scale increases with height, which might be due to horizontal entrainment and merging processes of the rising convective plumes. It is interesting that rotation also weakens this increase with height, and for run 4 the turbulent length scale is even nearly constant within the mixed layer.

A reduction of vertical entrainment at the top of the mixed layer has been found from other numerical experiments (Raasch and Etling 1991; Julien et al. 1996b) and is confirmed by our data. In Fig. 8 the kinematic heat flux normalized with its surface value is plotted against $z / h$. It is split into two parts: a resolved scale part caused by the turbulent eddies resolved by the model and a parameterized subgrid-scale part (dashed lines in Fig. 8). Due to the nature of LES models the latter should always be small compared to the resolved scale flux; this holds true except for the near-surface region where vertical transport must be done by smaller eddies that cannot be resolved by the numerical grid. Since the turbulent length scale is decreased with increasing rotation, the subgrid-scale transport must also be increased because heat transport is now done by smaller eddies. This is confirmed by Fig. 8. Nevertheless, the requirement stated above still holds for all model runs, although test runs show that it is no longer valid if the rotation rate is twice as large as in run 4 . In such cases the computational grid has to be refined. Figure 8 also confirms that the entrainment at the top of the mixed layer is significantly reduced when rotation is increased. This should affect the time rate of change of the mixed layer height $h$ because the entrainment of warmer fluid from the stratified layer will be less effective.

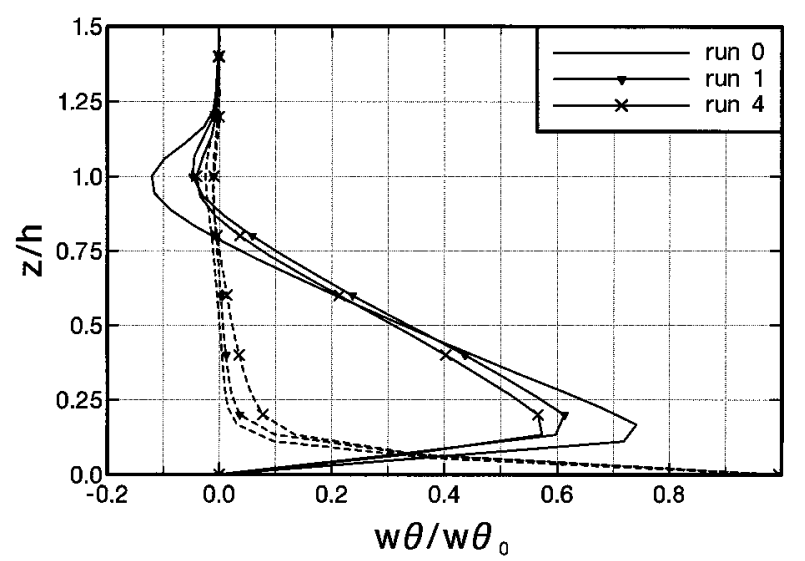

FIG. 8. A plot against nondimensional height of the resolved (solid) and subgrid-scale (dashed) kinematic heat flux for three model runs, normalized with the applied surface heat flux $w \theta_{0}$.

The mixed layer growth for all runs is shown in Fig. 9. Also included is the theoretical growth of a mixed layer without entrainment given by Eq. (2) for different ratios $B^{1 / 2} / N$ (solid lines). It is evident that the time rate of change of $h$ for early times of all rotational runs is only slightly larger than predicted by Eq. (2), which is reasonable because weak entrainment is still present. On the other hand, the mixed layer growth for the nonrotating run 0 is much larger compared to the rotating runs 1,4 , and 5 although they have the same ratio $B^{1 / 2} / N$. This effect of vertical entrainment can be included in Eq. (2) by using

$$
h_{t e}=2^{1 / 2}\left(B-B_{E}\right)^{1 / 2} t^{1 / 2} / N,
$$

where $B_{E}$ is the entrainment buoyancy flux (see, e.g., Stull 1988). Using (13) the predicted mixed layer height for the nonrotating run is about $10 \%$ larger and compares much better with the model result. Due to technical restrictions Coates et al. (1995) were not able to deter-

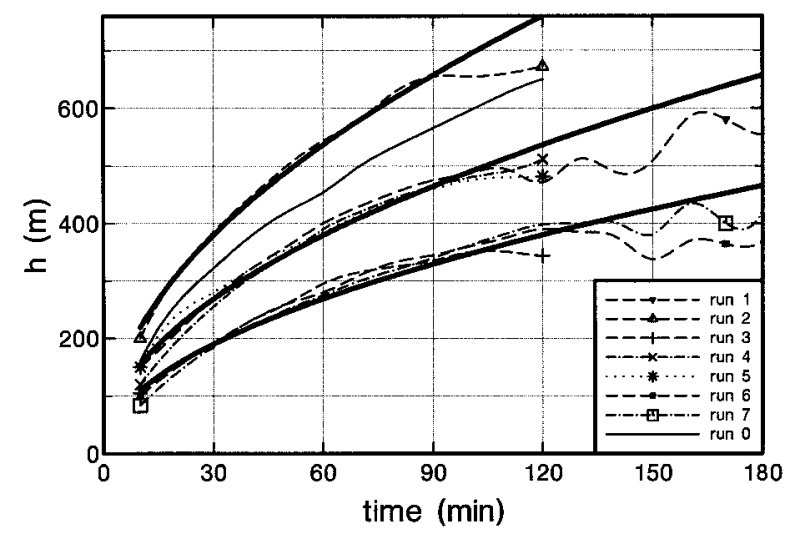

FIG. 9. The mixed layer height $h$ plotted against time. The thick solid lines show the theoretical mixed layer growth as computed from Eq. (2) for the combinations of buoyancy flux $B$ and Brunt-Väisälä frequency $N$ tested in the model runs. The thin solid line is from the nonrotating control run. 
mine mixed layer heights from the laboratory experiments, so they had to compute $h$ from Eq. (2) whenever they needed it in the analysis of the data. Our model results confirm that this method can be applied without making significant errors.

At later times the mixed layer growth for all rotational runs is slowed down or even disrupted. At this stage the surrounding ambient stratified fluid starts to horizontally mix into the convective mixed layer, which is an effect of the baroclinic eddies evolving from the breakdown of the rim current. Rim current and baroclinic eddies are analyzed in the next section. Unexpectedly, Fig. 9 indicates that the time $t=t_{s}$ when the mixed layer growth starts to slow down is not significantly controlled by rotation and is only a function of the buoyancy flux and the stratification in a way such that $t_{s}$ is small for those runs with a more rapid growth of the mixed layer. But this behavior of mixed layer growth has been also observed in recent experiments by Whitehead et al. (1996), who found that the final height of the mixed layer was independent of rotation rate. Theoretical considerations by Brickman (1995) and Visbeck et al. (1996) have led to the same conclusion. In short, the surface heating (cooling in the ocean) is compensated by horizontal heat flux at the periphery of the mixed layer due to baroclinic instability of the rim current. By this way, surface heating (cooling) does not lead to further heating (cooling) of the convective layer up to a greater depth.

\section{c. Rim current development and instability}

The rim current has been discussed already in section 4a and will now be investigated in detail for times before it becomes unstable. We tried to follow the rim-current analysis of Coates et al. (1995), who split the horizontal velocity data into radial $v_{r}$ and tangential $u_{\theta}$ components and normalized them by their expected velocity scale, yielding

$$
u_{\theta}^{*}=u_{\theta} /\left(B^{1 / 2} t^{1 / 2}\right) .
$$

Note that our velocity scale (11) derived in section 3 is smaller by a factor of 2 . Nevertheless, we used (14) for velocity scaling in order to allow for a better comparison between numerical and laboratory results. According to Coates et al. the radial coordinate is nondimensionalized by the local Rossby radius using (9) and (2), yielding

$$
R^{*}=\frac{R-R_{c}}{(2 B t)^{1 / 2} / f},
$$

where $R^{*}$ has been centered on the $\operatorname{rim} R_{c}$ of the heated circular surface.

Figure 10 shows the tangential velocity plotted against the radial distance for several heights. Data are from run 5 and for time $t=T$. Similar to the laboratory experiment, a Gaussian curve was fitted to this tangential velocity data. Coates et al. (1995) determined the approximate position $R_{\max }^{*}$ of this data and found the best-fit Gaussian curve for only those data beyond this point, which was necessary as the velocity data above the heated surface is influenced by the convective plumes. We proceed in the same way. The Gaussian fits are drawn as solid lines in Fig. 10 and as dashed lines when extrapolated into the region above the heated surface. The influence of the convective plumes represented by a large scatter in the data is confirmed by the numerical experiments. We also found a counterclockwise (cyclonic) rim current with distinct and persistent mean motions close to the surface (Fig. 10, $z / h=0.04$ ), which separates the mixed layer from a quiescent far field as well as a converse anticyclonic flow at greater heights (best seen in Fig. 10 at $z / h=0.60$ ).

The numerical runs show in addition to the laboratory experiment that these two counterrotating currents did not continuously turn into each other. A continuous change would imply that there must be a layer with zero tangential flow at about mid height of the mixed layer, which is not observed. Actually, the near-surface cyclonic rim current decreases with increasing height while the anticyclonic current becomes stronger, reaching its maximum in the upper half of the mixed layer. A first signal of this opposite current is a small distortion of the cyclonic rim current near the surface at $R^{*}=0$ where the tangential velocity has a relative minimum (Fig. 10, $z / h=0.12$ ). Higher above, both currents occur simultaneously. It was found difficult to fit a Gaussian curve for these situations, so it has been omitted in the corresponding plots (Fig. 10, $z / h=0.28$ ). The two currents occur simultaneously simply because they are a result of two different mechanisms acting at the same time. The cyclonic current is caused by the large-scale horizontal temperature gradient and can be well described by the thermal wind balance (10), which will be shown later in this section. It has a near-surface maximum because the radial temperature gradient above the rim of the heated surface increases continuously from $h$ toward the surface (see Fig. 3). The clockwise flow, on the other hand, is caused by an outflow of fluid at greater heights, which in turn is a reaction to frictionally forced surface inflow in order to obtain continuity. It can also be seen to be extending beyond the top of the mixed layer (Fig. 10, $z / h=1.07$ ), but with a much weaker amplitude. Again, this clockwise current can be explained by Eq. (10), since at those heights the temperature gradient is opposite to the near-surface gradient as discussed in section $4 \mathrm{a}$ and visible in Fig. 3.

From the Gaussian fits, the positions and magnitudes of the peak value of the rim current over all heights and for all runs were extracted and the peak current magnitude $u_{\text {peak }}^{*}$ plotted against the normalized height in Fig. 11. All values are taken at time $t=T$ in order to eliminate distortions of the rim current caused by the evolving baroclinic instabilities. As for the laboratory experiment, normalization of height is done by using $h$ as 

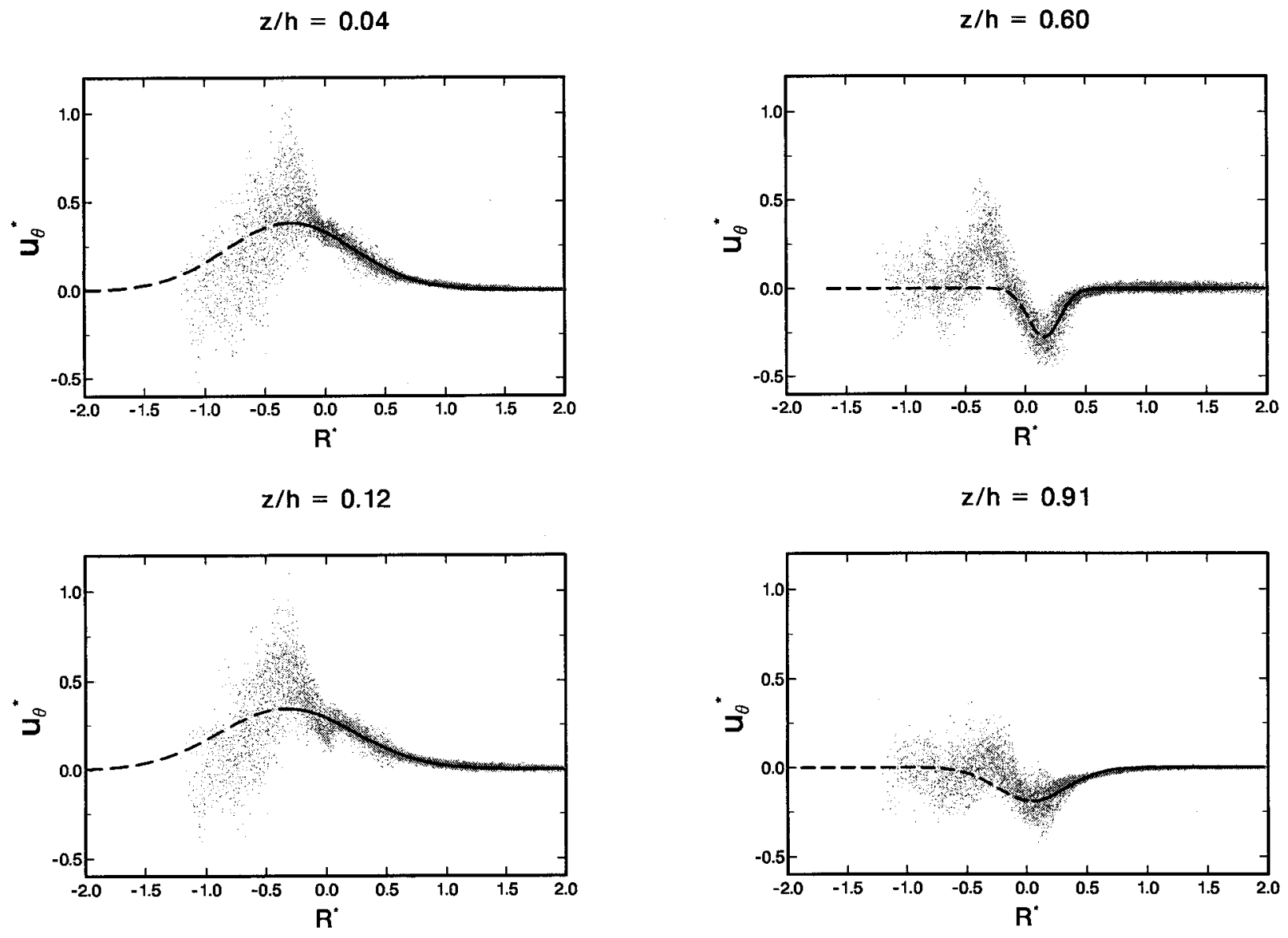

$z / h=0.28$

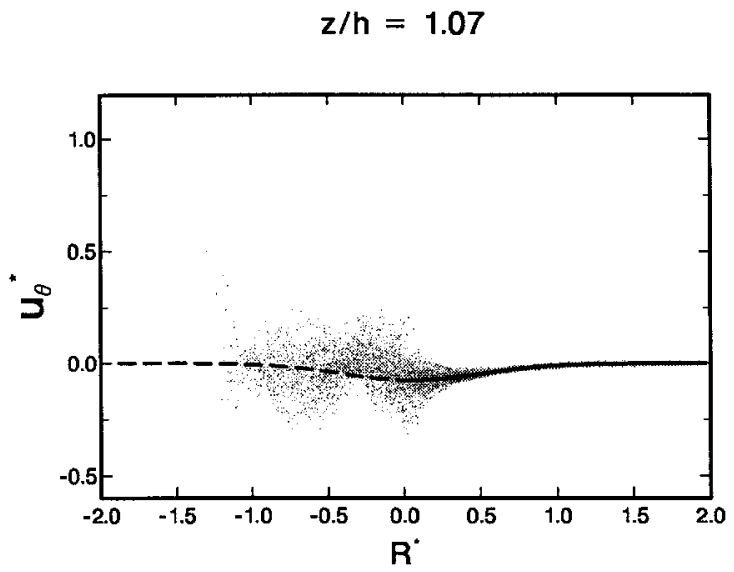

FIG. 10. The nondimensional tangential velocities $u_{\theta}^{*}$ at various heights for run 5 and at time $t=T$ plotted against the nondimensional distance $R^{*}$. The Gaussian fits are drawn as solid lines or as dashed lines when extrapolated into the region above the heated surface. It was found difficult to make a fit at nondimensional height $z / h=0.28$.

given by Eq. (2). Near the surface, the peak magnitude decreases linearly with height but there is, for some runs, a significant jump from positive to negative values at a height of about $0.3 \mathrm{~h}$, where for the first time the anticyclonic current becomes stronger than the near-surface cyclonic current. Nevertheless, a linear fit to all data within the mixed layer $(z / h<1)$ is

$$
u_{\text {peak }}=0.86 B^{1 / 2} t^{1 / 2}\left(0.47-\frac{z}{h}\right),
$$

drawn in Fig. 11 as a solid line, and it compares well with the fit from the laboratory data (dashed line in Fig. 11). Coates et al. (1995) stated that this overall linear decrease of the peak current magnitude is in agreement 


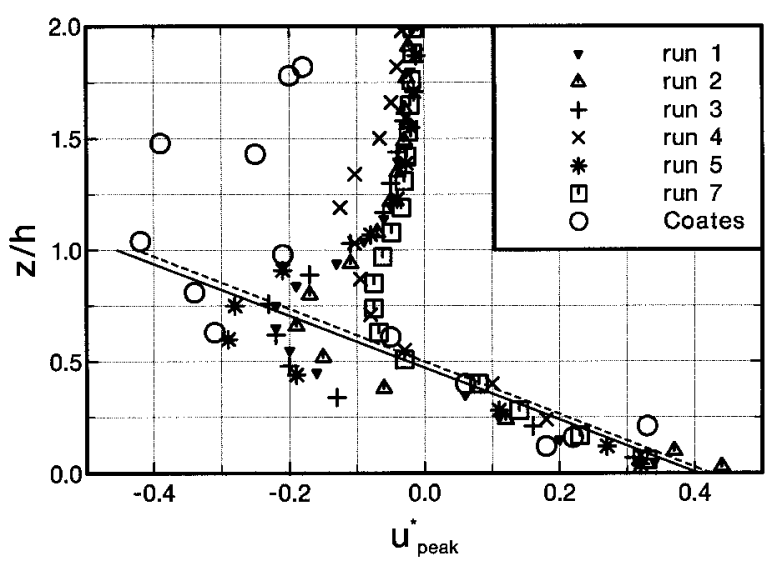

FIG. 11. The peak nondimensional tangential rim current velocity $u_{\theta}^{*}$ at time $t=T$ plotted against the nondimensional height. Laboratory results are marked by open circles. Also shown is the best fit to all data within the mixed layer $(z / h<1)$ for the laboratory experiment (Coates et al. 1995; dashed line) and the numerical runs (solid line).

with Eq. (10) when the radial temperature gradient is assumed constant with height. We would like to point out that Eq. (10) is unable to explain the large negative values of $u_{\text {peak }}$ since the radial temperature gradient below $z=h$ always has the same sign (even in the experiments where it is not constant but shows a linear increase from zero at $z=h$ toward the surface), and integration of (10) will result in an increase of rim current velocity from zero at $z=h$ to a maximum value at the surface.

Large negative values of $u_{\text {peak }}$ are not directly caused by the large-scale temperature gradient, as explained before, but are a consequence of the frictionally forced convergence of the near-surface cyclonic rim current. This is confirmed by Fig. 11 showing that negative peak velocities are largest for those runs with smallest rotation rates (e.g., run 5) where surface friction creates more horizontal flow convergence (see Fig. 4). Runs with large values of $f$ (runs 4 and 7), on the other hand, show only small negative peak velocities for heights above $0.5 h$. The laboratory data showed only a slow decay of the rim current velocity with height above the mixed layer (see open circles in Fig. 11), which was assumed to be produced by radiation of gravity waves and the penetrating convection. The numerical model, on the other hand, produced a strong decay of the velocity in this region for most of the runs, corresponding to the fact that the convective plumes indeed penetrate into the stable layer, however, not very far and in a random way that makes them unable to generate an organized circular current. The model shows that there is not much vertical propagation of momentum by subgrid-scale fluxes or gravity waves within the inversion and that the slight anticyclonic flow at these heights can be explained by the inverse radial temperature gradient. The horizontal outflow of fluid responsible for the strong anticyclonic flow only takes place below heights of

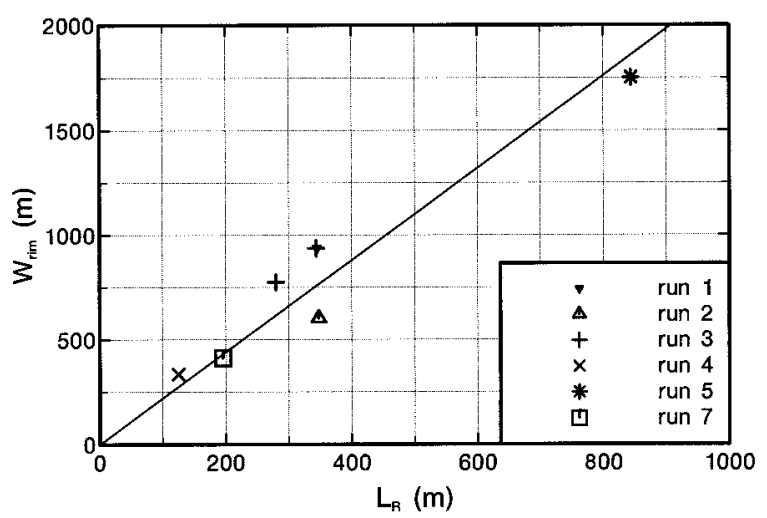

FIG. 12. Rim current width $w_{\text {rim }}=4 \sigma_{w}$ plotted against the local Rossby radius $L_{R}$ (all values taken at $t=T$ ). The solid line is the best fit to the numerical data.

$z \approx h$. We also performed two test runs with a minimum constant eddy viscosity (we choose values equal to 0.2 and $1.0 \mathrm{~m}^{2} \mathrm{~s}^{-1}$ respectively, which is the same order of magnitude compared to values resulting from the LES parameterization for the subgrid-scale turbulent eddy coefficients) in order to study if effects of molecular viscosity may be responsible for the strength of the counterrotating current within the stably stratified region. We found no significant influence on the structures and strength of the flow in this region. Generally, if molecular viscosity is strong enough to provide for an extension of the counterrotating current to greater depth, it should also act in horizontal directions leading to significant broadening of the current above the mixed layer. This was not observed in the laboratory experiment, so this specific difference between laboratory and numerical results still requires an explanation.

Coates et al. (1995) defined the rim current width $w_{\text {rim }}$ as $4 \sigma_{w}$, where $\sigma_{w}$ is the standard deviatian of the Gaussian fit. Using this definition, the near-surface rim current width from the numerical runs at time $t=T$ is plotted against the local Rossby radius (also taken at $t=T$ ) in Fig. 12. A linear fit to this data gives $w_{\text {rim }} \approx 2.2 L_{R}$, which compares quite well to the estimated laboratory value $w_{\text {rim }} \approx 2 L_{R}$ (although the laboratory data shows much more scatter) and confirms that the rim current width is clearly related to the Rossby radius.

Finally, we would like to discuss the instability of the rim current. Coates et al. (1995) had focused their analysis on a comparison between laboratory results and the results from the linear stability theory of Samelson (1993), although they found this comparison difficult due to the shortcomings already described in section 3 . In contrast to the assumptions made by linear stability theory, the base flow is unsteady during the experiments and, in particular, the mixed layer height; hence the local Rossby radius is increasing with time. Furthermore, the unsteady base flow is perturbed by the small-scale convective turbulence, which has a preferred length scale and therefore might control the wavelength of the in- 


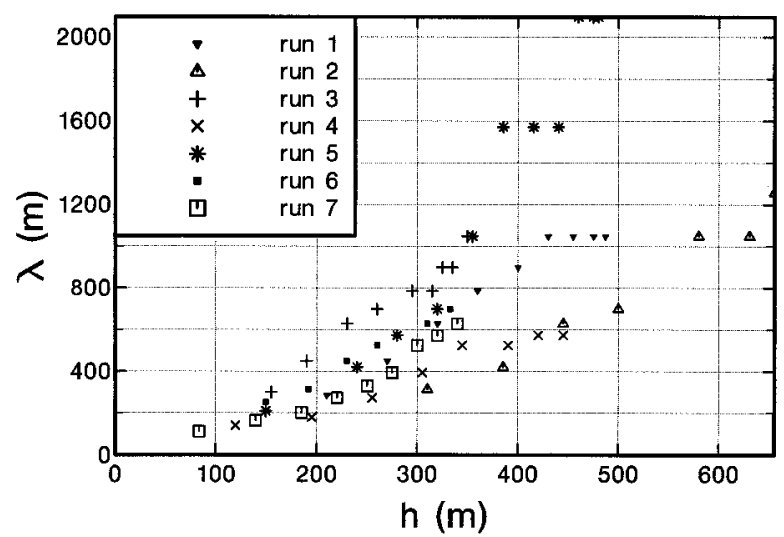

FIG. 13. The instability wavelength $\lambda$ plotted against the mixed layer height $h(t)$.

stability $\lambda$. Since this length scale is a function of the mixed layer height $h$ in the nonrotating scale (see section 4b), Coates et al. expected a dependence of $\lambda$ on $h$ but found this not to be confirmed by their data. However, Fig. 14 of Coates et al. may be at least interpreted in a way that $\lambda$ increases with increasing mixed layer height. The corresponding results from the numerical runs are plotted in Fig. 13, and the data shows a clear dependence $\lambda \sim h$, although significant scatter occurs. Since for rotating runs the integral length scale of the convective turbulence obviously depends on the rotation rate (section $4 b$ ), there should be an even stronger correlation between $\lambda$ and the local Rossby radius $L_{R}$. The numerical data presented in Fig. 14, indeed, show a good correlation between these two variables and a linear fit to the data gives $\lambda \approx 1.5 L_{R}$. Only run 5 shows larger variations from this fit, probably because surface friction is dominating over Coriolis force in this run. Since $L_{R}$ $\sim t^{1 / 2}$ according to Eqs. (9) and (2), Fig. 14 also indicates that the instability wavelength must increase with time, which is confirmed by the laboratory data and the tank experiments of Maxworthy and Narimousa (1994). After about four rotational periods the instability wavelength reached a maximum value and the waves break off into independent eddies, which slowly spread out filling the whole computational domain (data from this stage of evolution is not included in Fig. 14).

The maximum values of the instability wavelength $\lambda_{\max }$ at long times for the runs are plotted in Fig. 15 against the local Rossby radius at time $t=T$. This time has been chosen by Coates et al. (1995) since Samelson's (1993) analysis suggested that the $e$-folding time for the fastest growing wavelength is of order $1 T$; therefore they took the value of $h$ computed from Eq. (2) at $t=T$, using this estimate for this $h$ in calculating $L_{R}$. We proceed in the same way in order to make our data comparable to the analysis of the laboratory data. A linear fit to our data gives $\lambda_{\max } \approx 4.8 L_{R}$, shown in Fig. 15 as the solid line. This is quite different than $\lambda_{\max } \approx$ $3.3 L_{R}$ found from the laboratory data (dashed line in

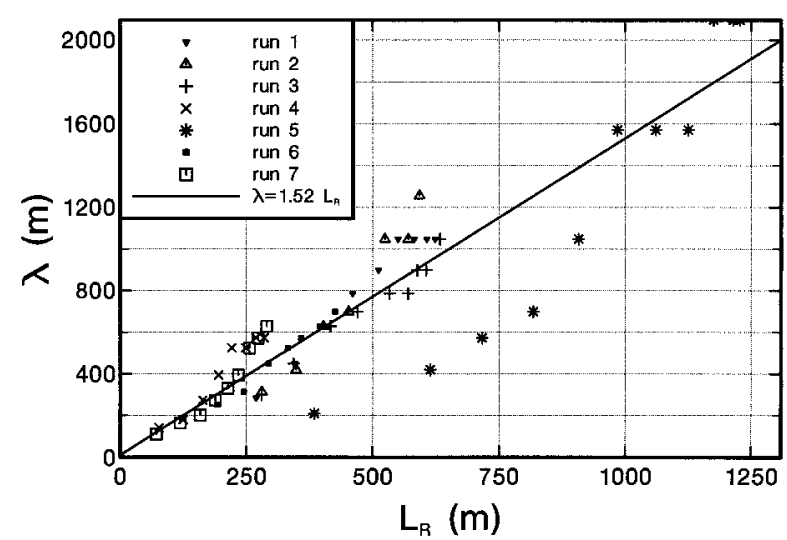

FIG. 14. The instability wavelength $\lambda$ plotted against the local Rossby radius $L_{R}(t)$. The solid line shows the best linear fit to the data.

Fig. 15) but compares quite well to Samelson's prediction from linear stability theory $\lambda_{\max } \approx 5 L_{R}$ for the fastest growing wavelength. Due to the problems in applying linear stability theory to our unsteady flow as described above, we are not sure if this result is only accidental. However, it is clear that theory, the laboratory experiment, and the numerical simulation are consistent in showing a dependence of the wavelength of the instability on the local Rossby radius. The growing baroclinic disturbances lead to a disruption of the convective deepening by horizontal mixing of the ambient fluid.

So far, the simulated properties of the rim current have been compared to laboratory experiments with a similar setup, that is, heating from below with a no-slip lower boundary. As already mentioned, for oceanic application cooling from above with a free-slip upper boundary would be more appropriate. This kind of simulation with localized heat sources has been performed, for example, by Jones and Marshall (1993), Send and Marshall (1995), and Sander et al. (1995). Although

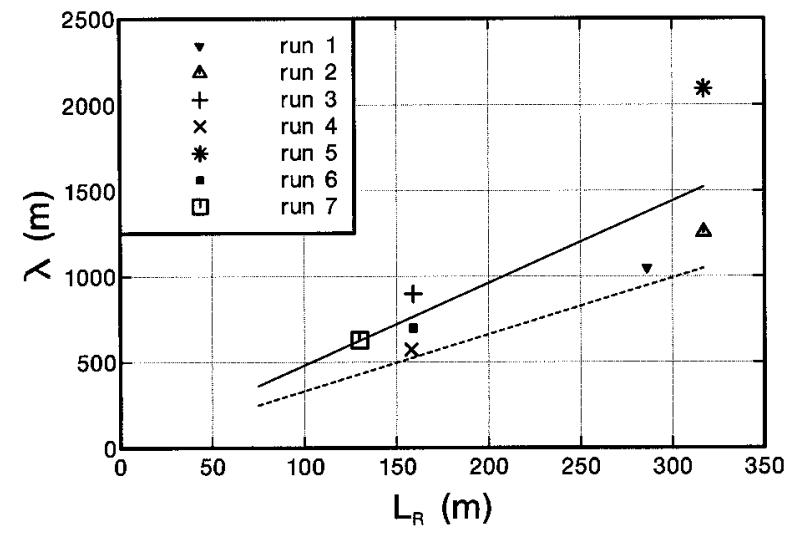

FIG. 15. The maximum instability wavelength from each run plotted against the local Rossby radius $L_{R}(t=T)$. The solid line is the best fit to the numerical data, while the dashed line shows the best fit to the laboratory data of Coates et al. (1995). 
most of their model runs were performed for a homogeneous ocean and with different numerical models (e.g., constant eddy viscosity), the structure of the rim current, including its final breakup into vortices are found to be quite similar to our model results. Also the scaling laws for rim current velocity, current width, and wavelength of the instabilities as analyzed in detail by Send and Marshall (1995) are closely related to the data presented here. This indicates that the formation of the rim current and its final breakup into vortices seems to be a quite robust phenomenon of rotating convection induced by a localized heat source and does depend only in detail on the numerical models and on the environmental conditions (e.g., homogeneous versus stratified fluid, no-slip, or free-slip boundaries).

\section{d. Application to the ocean}

Up until now our results have been compared essentially with the laboratory experiments of Coates et al. (1995), which was the main intention in order to verify our numerical model. But, as was mentioned in the introduction, various laboratory experiments and numerical simulations on rotating convection have been carried out in order to understand deep ocean convection. Coates et al. (1995) provide some comparison of their laboratory measurements with a number of field experiments in the Greenland Sea (Schott et al. 1993) and the Gulf of Lions (Leaman and Schott 1991; Schott et al. 1994). For these experiments they predicted mixed layer height, turbulent length and velocity scale, maximum rim current velocity, and the scale of the baroclinic eddies as they expect it from their laboratory results, and found that they are in general agreement with the field observations. However, they point out that such comparisons can only be approximative since detailed features of oceanic convection had not been considered in the laboratory experiments, such as the depth variation of the buoyancy frequency $N(z)$ for example. Similar comparisons with ocean data have been provided in the laboratory experiments by Maxworthy and Narimousa (1994) and Brickman and Kelly (1994).

The features observed in our numerical experiments should also be consistent with those observed in the field because they are in a good qualitative and quantitative correspondence with the laboratory experiments. Those model predictions showing large differences from the laboratory results, for example, the maximum instability wavelength $\lambda_{\max }$, are only hard to verify since detailed features of the ocean are not considered in the model and there is also a lack of field data of sufficient quality.

The simulations indicate that flow convergence due to surface friction is the main reason for the rim current occuring at mid height of the mixed layer. Since surface friction is present in the numerical model as well as in the laboratory experiment, but not at the boundary between the real atmosphere and ocean, this rim current rotating in opposite direction of the surface rim current should not be observed in the ocean.

Indeed numerical simulations of deep ocean convection by, for example, Send and Marshall (1995) and Sander et al. (1995) do not show this specific behavior of the rim current. But otherwise, properties like current speed, current width, and wavelength of instability are quite similar to our results, although the models differ, for example, with respect to subgrid turbulence (constant eddy viscosity versus LES-type closure). Hence, if we consider the numerical simulations mentioned above as a kind of oceanic dataset we may also conclude that our own model results should have some applications to deep ocean convection if scaled properly.

As an example, we compare some recent oceanic data for convective chimneys and rim currents obtained in the Gulf of Lions (Schott et al. 1996) and the Greenland Sea (Morawitz et al. 1996) with our numerical simulations. Schott et al. (1996) report a rim current in the field experiments of winter 1991/92 in the Gulf of Lions with a width of less than $20 \mathrm{~km}$ in a convective regime of $50-100-\mathrm{km}$ extent over a depth up to $1400 \mathrm{~m}$. Our model results would predict the size of the rim current as two times the Rossby radius as defined in (11). Taking a typical value $N=5 \times 10^{-3} \mathrm{~s}^{-1}$ in the area outside the convective chimney we obtain a Rossby radius of about $7 \mathrm{~km}$ and a rim current of about $15 \mathrm{~km}$, which is close to the observed size.

The breakup of the rim current due to baroclinic instability, as observed in the experiments by Coates et al. (1995) and the simulations presented here, could not be identified properly by Schott et al. (1996) because it could not be separated from the mesoscale variability in the flow field due to variable atmospheric forcing.

Observations in the Greenland Sea during winter 1988/89 by Morawitz et al. (1996) showed a convective chimney of about $50-\mathrm{km}$ extent and depth of more than $1000 \mathrm{~m}$. A rim current was not observed, but a breakup of the chimney within 3-6 days has been reported. We may compare this to our simulation, where the rim current broke up into vortices that moved away from the convective region within about four rotational periods or 4 days. It is difficult to compare more quantitative ocean data to the numerical experiments, as finescale observations of temperature and flow fields on scales ranging from individual convective plumes (a few hundred meters) to the chimney size (order of $10 \mathrm{~km}$ ) are still very difficult to obtain from oceanic field observations.

\section{Conclusions}

A number of numerical simulations were performed in order to study the processes of deep convection in a stratified ocean driven by a localized source of surface cooling. The simulations were conceived in a way that comparisons were possible with the recent laboratory experiments of Coates et al. (1995), which modeled deep 
convection as an inverted process in which convection is driven by heating the bottom of a rotating tank. The numerical experiments had been carried out with a largeeddy simulation model where subgrid-scale fluxes are parameterized using first-order closure, and eddy diffusivities are computed from the turbulence kinetic energy.

There is good qualitative correspondence between the numerical and the laboratory experiments. After the onset of convection above the heated surface a circular rim current begins to form between the convecting region and the surrounding ambient fluid due to the establishing horizontal temperature gradient between these two regions. After some time the growing rim current becomes unstable and breaks off into independent eddies, which spread out over the whole computational domain causing some horizontal exchange between the convective layer and the environment that in turn stops the mixed layer growth. This behavior was also found in experiments by Brickman (1995) and Whitehead et al. (1996) and was analyzed on theoretical grounds by Visbeck et al. (1996).

Similar to the laboratory experiments the simulations show that turbulent convection is not totally controlled by rotation and can be better explained by using nonrotational scaling rather than rotational scaling. Nevertheless, the simulations also show that convection is significantly affected by rotation. The turbulent length scale and the rms velocities within the mixed layer are smaller and the entrainment at the top of the mixed layer is less than in the nonrotating control run. For this last reason, the growth of the mixed layer can be described with a simple one-dimensional deepening law. All turbulent quantities have been directly determined from the simulation data, whereas in the laboratory some variables (like the turbulent length scale, for example) had only been estimated from other quantities. The entrainment was determined from the turbulent heat flux, which had not been measured in the laboratory experiment.

The rim current was analyzed with the same methods used in the laboratory. The modeled near-surface maximum of the rim current tangential velocity agrees well with the corresponding analytical solution given by integration of a simple thermal wind balance. Extending the laboratory analysis, the counterrotating current occuring at greater distances from the surface is explained by the near-surface convergence of the flow due to surface friction and probably will not be observed in the ocean. However, the rim current within the stable layer above the mixed layer that is also counterrotating with respect to the near-surface current can once again be explained by the horizontal radial temperature gradient at this height, which is opposite to the near-surface gradient. It is much weaker than observed in the laboratory.

The wavelength of the growing rim current instabilities clearly depends on the local Rossby radius, which is slowly increasing with time. The observed maximum wavelength is somewhat larger than in the laboratory experiment but compares quite well with the fastest growing wavelength predicted by linear stability theory. However, this correspondence may only be by chance since some of the main requirements of the linear theory are not met by the experiment. The break off into independent eddies takes place after about four rotational periods. The moment of disruption of the mixed layer growth was found to be dependent only on stratification of the ambient fluid and on the imposed surface buoyancy flux.

Laboratory data was found to be consistent with available field observations of deep ocean convection events. Since the observed features of the numerical runs are in good correspondence with the laboratory experiments, they should also correspond with the field observations. On the more general side, the detailed laboratory experiments by Coates et al. (1995) provide a comprehensive dataset for testing various numerical models on rotating convection that have been developed recently to study deep ocean convection.

\section{REFERENCES}

Adrian, R. J., R. T. D. Ferreira, and T. Boberg, 1986: Turbulent thermal convection in wide horizontal layers. Exp. Fluids, 4, 121141.

Bott, A., 1989: A positive definite advection scheme obtained by nonlinear renormalization of the advective fluxes. Mon. Wea. Rev., 117, 1006-1015.

Boubnov, B. M., and G. S. Golitsyn, 1990: Temperature and velocity field regimes of convective motions in a rotating plane fluid layer. J. Fluid Mech., 219, 215-239.

Brickman, D., 1995: Heat flux partitioning in open-ocean convection. J. Phys. Oceanogr., 25, 2609-2623.

_ _ and D. E. Kelley, 1994: Development of convection in a rotating fluid: scales and patterns of motion. Dyn. Atmos. Oceans, 19, 389-405.

Chlond, A., 1994: Locally modified version of Bott's advection scheme. Mon. Wea. Rev., 122, 111-125.

Coates, M. J., G. N. Ivey, and J. R. Taylor, 1995: Unsteady, turbulent convection into a rotating, linearly stratified fluid: Modeling deep ocean convection. J. Phys. Oceanogr., 25, 3032-3050.

Deardorff, J. W., 1972: Numerical investigation of neutral and unstable planetary boundary layers. J. Atmos. Sci., 29, 91-115.

, 1980: Stratocumulus-capped mixed layers derived from a threedimensional model. Bound.-Layer Meteor., 18, 495-527.

- and G. E. Willis, 1985: Further results from a laboratory model of the convective planetary boundary layer. Bound.-Layer Meteor., 32, 205-236.

Denbo, D. W., and E. D. Skyllingstad, 1996: An ocean large-eddy simulation model with application to deep convection in the Greenland Sea. J. Geophys. Res., 101, 1095-1110.

Fernando, H. J. S., and C. Y. Ching, 1993: Effects of background rotation on turbulent line plumes. J. Phys. Oceanogr., 23, 21252129.

_ R. Chen, and D. L. Boyer, 1991: Effects of rotation on convective turbulence. J. Fluid Mech., 228, 513-547.

Garwood, R. W., S. M. Isakari, and P. C. Gallacher, 1994: Thermobaric convection. The Polar Oceans, Geophys. Monogr., No. 85, Amer. Geophys. Union, 199-209.

Gascard, J. C., 1991: Open ocean convection and deep water formation revisited in the Mediterranean, Labrador, Greenland and Weddell Seas. Deep Convection and Deep Water Formation in the Oceans, P. C. Chu and J. C. Gascard, Eds., Elsevier Oceanogr. Ser., Vol. 57, Elsevier, 157-182. 
Hopfinger, E. J., and P. F. Linden, 1990: The effect of background rotation on fluid motions: A report on Euromech 245. J. Fluid Mech., 211, 417-435.

Hunt, J. C. R., 1984: Turbulence structure in thermal convection and shear-free boundary layers. J. Fluid Mech., 138, 161-184.

Ivey, G. N., J. R. Taylor, and M. J. Coates, 1995: Convectively driven mixed layer growth in a rotating, stratified fluid. Deep-Sea Res., 42, 331-349.

Jones, H., and J. Marshall, 1993: Convection with rotation in a natural ocean: A study of open ocean convection. J. Phys. Oceanogr., 23, 1009-1039.

Julien, K., S. Legg, J. McWilliams, and J. Werne, 1996a: Rapidly rotating turbulent Rayleigh-Bénard convection. J. Fluid Mech., 322, 243-273.

,,,--- , and,$- 1996 \mathrm{~b}$ : Penetrative convection in rapidly rotating flows: Preliminary results from numerical simulation. Dyn. Atmos. Oceans, 24, 237-249.

Killworth, P. D., 1983: Deep convection in the World Ocean. Rev. Geophys. Space Phys., 21, 1-26.

Klinger, B. A., and J. Marshall, 1995: Regimes and scaling laws for rotating deep convection in the ocean. Dyn. Atmos. Oceans, 21, $227-256$.

Lavelle, J. W., and D. C. Smith IV, 1996: Effects of rotation on convective plumes from line segment sources. J. Phys. Oceanogr., 26, 863-872.

Leaman, K. D., and F. A. Schott, 1991: Hydrographic structure of the convective regime in the Gulf of Lions: Winter 1987. J. Phys. Oceanogr., 21, 575-598.

Madec, G., M. Chartier, P. Delecluse, and M. Crepon, 1991: A threedimensional numerical model of deep-water formation in the northwestern Mediterranean Sea. J. Phys. Oceanogr., 21, 13491371.

Maxworthy, T., and S. Narimousa, 1994: Unsteady turbulent convection into a homogeneous, rotating fluid, with oceanographic applications. J. Phys. Oceanogr., 24, 865-887.

Morawitz, W. M. L., P. J. Sutton, P. F. Worcester, and B. D. Cornuelle, 1996: Three-dimensional observations of a deep convective chimney in the Greenland sea during winter 1988/89. J. Phys. Oceanogr., 26, 2316-2343.
Narimousa, S., 1996: Penetrative turbulent convection into a rotating two-layer fluid. J. Fluid Mech., 321, 299-313.

Nieuwstadt, F. T. M., P. J. Mason, C.-H. Moeng, and U. Schumann, 1991: Large-eddy simulation of the convective boundary layer: A comparison of four computer codes. Turbulent Shear Flows, F. Durst et al., Eds., Vol. 8, Springer Verlag, 343-367.

Raasch, S., and D. Etling, 1991: Numerical simulation of rotating turbulent thermal convection. Beitr. Phys. Atmosph., 64, 185199.

Samelson, R. M., 1993: Linear instability of a mixed-layer front. $J$. Geophys. Res., 98, 10 195-10 204.

Sander, J., D. Wolf-Gladrow, and D. Olbers, 1995: Numerical studies of open ocean deep convection. J. Geophys. Res., 100, 20 57920600 .

Schmidt, H., and U. Schumann, 1989: Coherent structure of the convective boundary layer derived from large-eddy simulations. $J$. Fluid Mech., 200, 511-562.

Schott, F. A., M. Visbeck, and J. Fischer, 1993: Observations of vertical currents and convection in the Greenland Sea during the winter of 1988/89. J. Geophys. Res., 98, 14 401-14 422.

- - - , and U. Send, 1994: Open ocean convection, Mediterranean and Greenland Seas. Ocean Proc. Climate Dyn., C149, 203-225.

, J. Fischer, L. Stramma, and Y. Desaubies, 1996: Observations of deep convection in the Gulf of Lions, northern Mediterranean, during winter of 1991/92. J. Phys. Oceanogr., 26, 505-524.

Send, U., and J. Marshall, 1995: Integral effects of deep convection. J. Phys. Oceanogr., 25, 855-872.

Stull, R. B., 1976: Mixed layer depth model based on turbulent energetics. J. Atmos. Sci., 33, 1268-1278.

- 1988: An Introduction to Boundary Layer Meteorology. Kluwer Academic, $666 \mathrm{pp}$.

Visbeck, M., J. Marshall, and H. Jones, 1996: Dynamics of isolated convective regions in the ocean. J. Phys. Oceanogr., 26, 17211734 .

Whitehead, J. A., J. Marshall, and G. E. Hufford, 1996: Localized convection in rotating stratified fluid. J. Geophys. Res., 101, $25705-25721$. 